Table 1. Uno Concordance Scores. Format 'mean (min-max)'

\begin{tabular}{lcccc}
\hline & & Baseline & Week 4 & Week 8 \\
\hline Cox Proportional Hazards & Change 1 & $0.63(0.59-0.69)$ & $0.72(0.68-0.80)$ & $0.75(0.69-0.82)$ \\
& Change 2 & $0.58(0.50-0.66)$ & $0.75(0.65-0.87)$ & $0.78(0.65-0.93)$ \\
Linear Multi-Task Regression & Change 1 & $0.65(0.62-0.68)$ & $0.72(0.68-0.78)$ & $0.75(0.69-0.79)$ \\
& Change 2 & $0.58(0.51-0.68)$ & $0.72(0.67-0.82)$ & $0.77(0.68-0.89)$ \\
Random Survival Forest & Change 1 & $0.64(0.58-0.67)$ & $0.71(0.66-0.78)$ & $0.76(0.68-0.80)$ \\
& Change 2 & $0.56(0.50-0.68)$ & $0.72(0.60-0.82)$ & $0.77(0.64-0.92)$ \\
\hline
\end{tabular}

Conclusion: Our data-driven approach identified predictive clinical factors with a high face validity including joint counts, functionality scores and global health indicators. The different model approaches did not seem to increase the predictive capacity performance. However, our results underline that not so much the baseline disease status but rather the early response to initial treatment reflected in the selected predictive factors can be used for prediction of the need for further treatment adaptation. These models will have to be enriched with patient reported outcomes to further improve predictive performance.

Disclosure of Interests: None declared

DOI: 10.1136/annrheumdis-2021-eular.2717

\section{POS1435 CLINICAL CHARACTERIZATION AND PREDICTIVE FACTORS FOR PROGRESSION IN A COHORT OF ILD PATIENTS WITH FEATURES OF AUTOIMMUNITY: ARE IPAF CRITERIA SUFFICIENT?}

F. Bozzao ${ }^{1}$, P. Tomietto ${ }^{2}$, E. Baratella ${ }^{3,4}$, F. Giudici $^{4}$, M. Kodric ${ }^{5}$, R. Della Porta ${ }^{5}$, I. Prearo ${ }^{6}$, S. M. G. Pirronello ${ }^{7}$, F. Fischetti ${ }^{2,4}$, M. Confalonieri ${ }^{4,5}$, B. Fabris ${ }^{4,8}$ on behalf of ILD multidisciplinary unit of the "Azienda sanitaria universitaria Giuliano Isontina" (Trieste). 'Azienda ULSS 2 Marca Trevigiana, Internal Medicine, Treviso, Italy; ${ }^{2}$ Azienda Sanitaria Universitaria Giuliano Isontina, Internal Medicine - Rheumatology Unit, Trieste, Italy; ${ }^{3}$ Azienda Sanitaria Universitaria Giuliano Isontina, Radiology, Trieste, Italy; ${ }^{4}$ University of Trieste, Medicine, Surgery and Health Sciences, Trieste, Italy; ${ }^{5}$ Azienda Sanitaria Universitaria Giuliano Isontina, Pneumology, Trieste, Italy; ${ }^{6}$ University Hospital, LMU Munich, Vascular Medicine, Munich, Germany; ${ }^{7}$ Azienda Ospedaliera Cannizzaro, Emergency Medicine, Catania, Italy; ${ }^{8}$ Azienda Sanitaria Universitaria Giuliano Isontina, Internal Medicine, Trieste, Italy

Background: It is unknown whether patients with interstitial lung disease (ILD) and only some features of autoimmunity have a different natural history from those with a defined connective tissue disease (CTD-ILD). The classification criteria for "ILD with autoimmune features" (IPAF) may not be able to characterize all these patients, especially those with a usual interstitial pneumonia (UIP) pattern [1].

Objectives: To determine clinical characteristics and predictive factors for progression in a cohort of ILD patients with features of autoimmunity, through the application of classification criteria for IPAF and specific CTD, whenever possible.

Methods: We retrospectively selected a cohort of consecutive patients with ILD as onset manifestation and features of autoimmunity (at least 1 autoantibody and/or 1 clinical sign/symptom), evaluated by our multidisciplinary unit from March 2009 to March 2020. All the final diagnoses were revised according to the latest CTD and IPAF criteria. Patients were followed up for 33 (16.5-69.5) months.

Results: Of the 101 patients enrolled (67.4 $\pm 10.9 \mathrm{yrs}, \mathrm{F} / \mathrm{M}$ ratio $65 / 36), 53(52.5 \%)$ and $37(36.6 \%)$ respectively satisfied the CTD and IPAF criteria. Eleven patients (10.9\%) did not satisfy IPAF criteria because of only 1 item (clinical or serologic) within the IPAF domains and a UIP pattern; we defined this group as "autoimmune" UIP (AI-UIP). All the 8 patients initially classified as undifferentiated CTD had sufficient IPAF criteria. Among the IPAF patients $(68.2 \pm 10.1$ years, F/M ratio 20/17), the most common findings were: Nonspecific interstitial pneumonia pattern $(56.8 \%)$, antinuclear antibodies positivity $(43.2 \%)$ and arthritis $(24.3 \%)$. The combination of a positive morphologic and serologic domain was the most common to reach the diagnosis $(48.6 \%)$. Some IPAF patients had features not included in IPAF criteria, such as non-anti-synthetase myositis-specific antibodies $(21.6 \%)$, objective sicca syndrome $(13.5 \%)$ and anti-myeloperoxidase antibodies $(2.7 \%)$. Over a median of 17 months, 2 IPAF patients $(5.4 \%)$ developed a definite UIP pattern, while $4(10.8 \%)$ a specific CTD. Comparing the IPAF, CTDILD and AI-UIP groups, no statistically significant differences were found in the mean age, sex distribution, smoking habits and mean duration of the disease. However, IPAF patients had a significantly higher prevalence of arterial hypertension and left-sided heart failure and a lower predominance of UIP pattern as expected $(10.8 \%$ vs. $32.1 \%$ vs. $100 \%, p<0.01)$. Although no differences were found at the diagnosis, at 1 year the proportion of IPAF patients with radiological progression of the fibrosis and/or functional deterioration (defined by a decline in FVC of $\geq 10 \%$ and/or DLCO of $\geq 15 \%$ predicted) was lower to that of CTD-ILD and AI-UIP (17.1\% vs. $31.4 \%$ vs. $63.6 \%, p$ 0.01). Fewer IPAF patients needed oxygen support ( $8.6 \%$ vs. $31.4 \%$ vs. $36.4, p$ 0.02). Considering the overall 101 patients, having an IPAF and a UIP pattern respectively predicted a slower (OR $0.37, p 0.04$ ) and a faster (OR: $3.56, p$ 0.01) ILD progression at the multivariate analysis.

Conclusion: In our cohort, IPAF criteria were useful to identify a subset of patients with a slower ILD progression and a possible evolution to CTD (10$15 \%$ of cases) [2]. These criteria do not characterize all the patients with a UIP pattern and limited features of autoimmunity, which seem to have a worse prognosis, independently from the final diagnosis. Further studies are needed to clarify if the prognosis of AI-UIP is different from that of idiopathic pulmonary fibrosis.

\section{REFERENCES:}

[1] Graney, et al. Ann Am Thorac Soc 2019;16(5):525-33.

[2] Sebastiani, et al. Biomedicines 2021,9,17.

DISCLOSURE OF INTERESTS:NONE DECLARED

DOI: 10.1136/annrheumdis-2021-eular.2899

\section{POS1436 EPIDEMIOLOGY OF LATENT TUBERCULOSIS INFECTION IN PATIENTS WITH RHEUMATIC IMMUNE- MEDIATED DISEASES. SINGLE UNIVERSITY STUDY OF 1117 PATIENTS}

D. Martínez-López ${ }^{1}$, J. Osorio-Chavez ${ }^{2}$, C. Álvarez-Reguera ${ }^{3}$, V. Portilla ${ }^{3}$, M. A. González-Gay ${ }^{3}$, R. Blanco ${ }^{3} .{ }^{1}$ Hospital Universitario Marqués de Valdecilla, Rheumatology, Santander (SPAIN), Spain; ${ }^{2}$ Hospital Universitario Marqués de Valdecilla, Pulmonology, Santander (SPAIN), Spain; ${ }^{3}$ Hospital Universitario Marqués de Valdecilla, Rheumatology, Santander, Spain

Background: Patients with rheumatologic immune-mediated diseases (R-IMID) with Latent tuberculosis infection (LTBI) requiring biologic therapy (BT) are at an increased risk of active tuberculosis (TB). Screening of LTBI with tuberculin skin test (TST) and/or Interferon (IFN)- $\gamma$ release assays (IGRA) is recommended before starting of BT.

Objectives: In patients with R-IMID previously to BT our aim was to assess a) prevalence of $\mathrm{LTBI}, \mathbf{b}$ ) importance of using a booster test in negative TST and $\mathbf{c}$ ) to compare TST with the IGRA test.

Methods: Cross-sectional single University Hospital study including all patients diagnosed with R-IMID who underwent a TST and/or IGRA in the last five years (2016-2020).

TST was performed by a subcutaneous injection of $0.1 \mathrm{ml}$ of purified protein derivative (PPD) with a reading after 72 hours. TST was considered positive with an induration of more than $5 \mathrm{~mm}$ of diameter. If the first TST was negative, a new TST (Booster) was performed between 1 and 2 weeks after the first TST.

LTBI was diagnosed by a positive IGRA and/or TST and absence of active TB (Chest radiograph). Diagnosis with IGRA vs TST was compared (Cohen's kappa coefficient)

Results: We included 1117 patients ( 741 women/376 men), mean age $53 \pm 15$ years with LTBI. Chest radiograph was normal in most of the patients, only 39 patients $(3.5 \%)$ presented signs of previous TB infection, mostly granuloma Total LTBI prevalence was $31.7 \%$ (354/1117). LTBI prevalence in different underlying R-IMID ranges from $35 \%$ in vasculitis up to $26.5 \%$ in conectivopathies (Figure 1).

Booster was positive in 66 patients (7.7\%) out of 859 patients with a negative simple TST. Results of TST (+booster) and IGRA tests are shown in Table 1. TST (+booster) was positive in 187 patients $(22.9 \%)$ out of 817 with a negative or indeterminate IGRA test. IGRA test was positive in $30(3.8 \%)$ out of 793 patients with a negative TST (+booster). Cohen's Kappa coefficient between TST (+booster) and IGRA (QFT-plus), was 0.381.

Conclusion: LTBI is frequent between patients with R-IMID. Booster after negative simple TST may be useful, since it can detect false negatives for LTBI. IGRA and TST(+booster) show a low grade of agreement. Therefore, performing both tests before BT may be recommendable.

Table 1. Results of TST (+booster) and IGRA test

\begin{tabular}{lcccccc}
\hline \multicolumn{7}{c}{ IGRA (QFT-Plus) } \\
\hline & & Positive & Negative & Indeterminate & Unavailable & Total \\
\hline TST & Positive & 89 & 142 & 45 & 48 & 324 \\
& Negative & 30 & 500 & 130 & 133 & 793 \\
(+Booster) & Total & 119 & 642 & 175 & 181 & 1117 \\
& & & & & & \\
\end{tabular}

* Cohen's kappa coefficient: 0.381 
Total $\mathbf{n}$ LTB

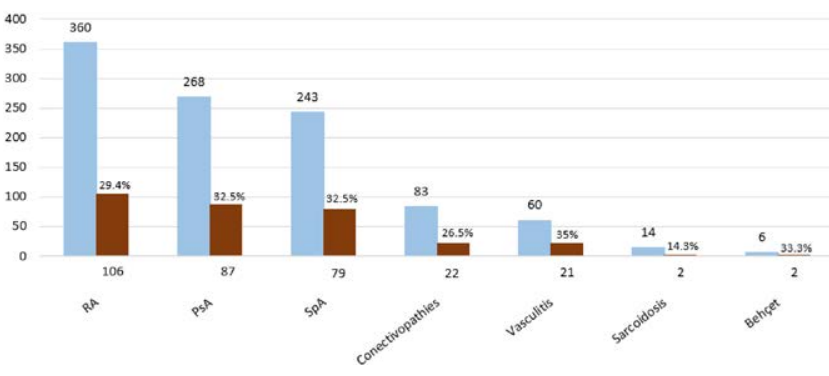

Figure 1. Prevalence of LTBI in different underlying R-IMID

LTBI: Latent tuberculosis infection, PsA: Psoriatic arthritis, RA: Rheumatoid arthritis, SpA: Axial spondyloarthritis.

Diagnosis of LTBI: Positive TST(+booster) and/or IGRA test.

Disclosure of Interests: David Martínez-López: None declared, Joy Osorio-Chavez: None declared, Carmen Álvarez-Reguera: None declared, Virginia Portilla: None declared, Miguel A González-Gay Speakers bureau: Abbvie, Pfizer, Roche, Sanofi and MSD, Consultant of: Abbvie, Pfizer, Roche, Sanofi and MSD, Grant/research support from: Abbvie, MSD, Jansen and Roche, Ricardo Blanco Speakers bureau: Abbvie, Pfizer, Roche, Bristol-Myers, Janssen, Lilly and MSD, Consultant of: Abbvie, Pfizer, Roche, Bristol-Myers, Janssen, Lilly and MSD, Grant/research support from: Abbvie, MSD, and Roche DOI: 10.1136/annrheumdis-2021-eular.2900

\section{POS1437 LATENT TUBERCULOSIS INFECTION IN RHEUMATIC DISEASES: A REAL-LIFE STUDY OF THREE APULIAN CENTRES. DATA FROM BIOPURE REGISTRY}

M. Fornaro ${ }^{1}$, D. Goletti ${ }^{2}$, A. Abbruzzese ${ }^{1}$, M. G. Anelli ${ }^{1}$, A. Semeraro ${ }^{3}$, N. Maruotti ${ }^{4}$, F. P. Cantatore ${ }^{4}$, F. Cacciapaglia ${ }^{1}$, F. lannone ${ }^{1}$ on behalf of BIOlogic aPUlian REgistry (BIOPURE). ${ }^{1}$ Rheumatology Unit, Department of Emergence and Transplantation (DETO), University of Bari, Bari, Italy; ${ }^{2}$ Translational Research Unit, Department of Epidemiology and Preclinical Research, National Institute for Infectious Diseases, L. Spallanzani, Roma, Italy; ${ }^{3}$ Rheumatology Unit, ASL Taranto, Taranto, Italy; ${ }^{4}$ UOC Reumatologia Universitaria, University of Foggia, Foggia, Italy

Background: Latent tuberculous infection (LTBI) is very common in the world and screening for it is essential before starting treatment with biotechnological drugs

Objectives: The aims of our study were to assess the prevalence in Apulia of LTBI among patients affected with rheumatic disease and to record the cases of tuberculosis (TB) infection among patients treated with biologic agents.

Methods: We analysed data of patients included in BIOPURE registry from 2008 to 2018, who underwent Quantiferon (QTF) test as routinely screening for biologic treatment. Demographic and clinical data were recorded at the time of the first QTF assessment and this time point was considered the "baseline" of the study. Data regarding further QTF tests performed during follow-up was also acquired by electronic charts. Prophylaxis administration and bDMARD treatments were recorded for patients with positive QTF test. All tuberculosis infections were recorded during the entire time of follow-up.

Results: Three thousand thirty-five patients (female $67.2 \%$, mean age $52 \pm 18.3$ years) were included in these study, 2692 patients $(88.7 \%)$ had inflammatory arthritis $(28.2 \%$ rheumatoid arthritis, $33 \%$ psoriatic arthritis and $27.4 \%$ spondyloarthritis), 129 (4.2\%) patients had connective tissue disease, whereas 214 $(7.1 \%)$ patients were affected by others rheumatic diseases. The prevalence of LTBI was 10.7\% (326 patients) at baseline. Comparisons between positive and negative patients for QTF are reported in Table 1. We acquired data of LTBI prophylaxis of 284 patients; 235 out 265 patients treated with isoniazid completed the treatment, whereas 19 out 19 patients treated with rifampicin completed the prophylaxis regimen. The main cause of isoniazid withdrawal was hypertransaminasemia, but 8 patients then completed prophylaxis with rifampicin. During the entire follow-up $(42.6 \pm 30.5$ months), we recorded 5 $(0.02 \%)$ cases of primary TB infection in patients on anti-TNF $\alpha$ agents treatment, which had baseline screening negative for LTBI. Data and outcome of these patients are reported in Table 2. The mean time of follow-up of patients on bDMARDs treatment with positive QTF at baseline was $52.7 \pm 35.2$ months. bDMARD treatment regimens are reported in Table 3. No case of TB reactivation was found among patients with positive baseline QTF. Moreover, of 1563 (51.5\%) patients who repeated QTF during follow-up, $62(4 \%)$ of them showed a change in the test result. We observed a change to a positive state in 36 patients with previous negative QTF test, whereas 26 patients with previous positive QTF showed a shift to a negative test during follow-up.

Conclusion: Our study shows a prevalence of LTBI of $10.7 \%$ in Apulian patients affected with rheumatic disease. bDMARDs therapy appears to be safe in patients with positive QTF test treated according to current recommendations ${ }^{1}$. However, cases of primary TB infections, especially in patients receiving antiTNF $\alpha$ drugs, have been observed.

REFERENCES:

[1] Cantini F, et al, Guidance for the management of patients with latent tuberculosis infection requiring biologic therapy in rheumatology and dermatology clinical practice, Autoimmun Rev (2015).

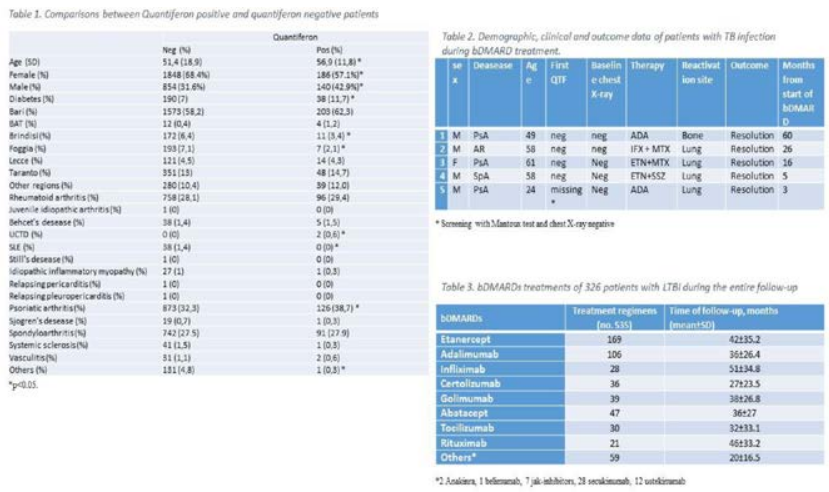

Disclosure of Interests: None declared

DOI: 10.1136/annrheumdis-2021-eular.2932

\section{POS1438 EARLY FACTORS ASSOCIATED WITH THE INITIATION OF TREATMENT WITH BIOLOGICS IN PATIENTS WITH AXIAL SPONDYLOARTHRITIS - RESULTS FROM A SINGLE CENTRE RETROSPECTIVE COHORT STUDY}

E. Costa ${ }^{1,2}$, L. Lages ${ }^{3}$, D. Esperança Almeida ${ }^{1,2}$, A. M. Gomes Correia ${ }^{1}$, M. Cerqueira ${ }^{1}$, J. Silva ${ }^{1}$, A. R. Ribeiro ${ }^{1}$, J. Redondo Costa ${ }^{1}$, J. Neves ${ }^{1} .{ }^{1}$ Hospital de Braga, Rheumatology, Braga, Portugal; ${ }^{2}$ Unidade Local de Saúde do Alto Minho, Rheumatology, Ponte de Lima, Portugal; ${ }^{3}$ University of Minho, School of Medicine, Braga, Portugal

Background: Axial Spondyloarthritis (axSpA) refers to a group of rheumatic dis eases that mainly affect the axial skeleton. Treatment with Biological Disease Modifying Anti-Rheumatic Drug (bDMARDs) is indicated when low disease activity is not achieved with Non-Steroid Anti-inflammatory Drugs. Some studies reported that some clinical and socio-demographic were associated with biologics initiation ${ }^{1,2}$. However, early factors associated with the initiation of bDMARDs have not been extensively studied.

The authors speculate that certain features early in disease presentation may be associated with future need for treatment with bDMARDs in patients with axSpA. Objectives: To study a population of patients with axSpA and determine whether the presence of certain factors at diagnosis is associated with a later need for biological treatment.

Methods: A single centre retrospective study was conducted comprising patients fulfilling the ASAS criteria for axSpA that attended the Rheumatology Outpatient Clinic from January to December 2019. Patients with psoriatic arthritis and enteropathic spondyloarthritis were excluded. All data, except regarding use of bDMARD, refers to the two initial appointments. Between group comparisons were performed through independent sample t-test or Chi-Square as applicable. Logistic Multivariate Regression was performed to understand which factors independently contributed to the use of bDMARDs.

Results: One hundred and fifty patients were included, and 52 patients $(34,7 \%)$ were under biological treatment. In comparison to the group that was not under treatment with bDMARDs, these were significantly more likely to be hard-workers $(57,8 \%$ vs $29,7 \% ; p=, 003)$, to have had elevated C-Reactive Protein $(81,6 \%$ vs $48,9 \%$; $p<, 001)$, to have had a grade of sacroiliitis greater than $2(67,4 \%$ vs $29,5 \% ; p<, 001)$ and to have history of enthesitis, $(32,7 \%$ vs $13,3 \% ; p=, 006)$. No differences between groups were found in sex, age, HLA-B27 positivity, state of employment, diagnostic delay, smoking status, history of dactylitis and history of uveitis. In multivariate regression analysis, only the hard-worker type and the highest grade of sacroiliitis were found to be independently associated with the use of bDMARDs as presented in Table 1. Although not statistically significant, a trend to association was found between elevated CRP at diagnosis and use of bDMARDs. 